The secondary endpoint was to analyze the characteristics of patients who remain for a longer period of time in an optimized dose regimen.

Methods: In our Rheumatology Unit we are treating 271 RA patients with BT, the dose was deintensificated for $62(23 \%)$ patients in remission or low disease activity for at least 6 months. We have selected 32 patients with BT reduced for at least 6 years in an observational, descriptive, longitudinal and retrospective study. Disease activity was measured by the DAS 28 index. Structural damage was evaluated by SENS method.

Results: We analyze 32, 20 female, 12 male, mean age at diagnosis 42.6 years old; BT was started after RA evolution of 98.63 months. Drug reduction was performed after full BT for 62 months, mean DAS 28 was 2,47.

Patients were $75 \%$ FR positive and $56.7 \%$ ACPA positive. Etanercept was the BT more commonly reduced $59.4 \%$, followed by adalimumab $21.9 \%$, infliximab $12.5 \%$ and certolizumab $6.3 \%$.

BT dose returned to normal for 11 patients because of disease activity worsening after an average time of 15.90 months.

For 21 patients remaining on reduced doses, the mean DAS28 at time for analysis was 2.67.

BT reduction as different drugs: none infliximab reduced dose patients required return to normal dose. All certolizumab reduced (2 patients) patients needed to back to normal dose. Etanercept in 36,8\% and adalimumab 28,6\%.

The mean of SENS score before the optimization was 8.78 and at time for analysis 10.67 for both kind of patients, who continued reduced and those who needed to increase BT dose.

For the secondary endpoint 10 out of 12 male continue with deintensificated BT $(83 \%)$ in the other hand only 11 out of 20 female $(55 \%)$ maintained reduced dose.More negative for FR $(69,2 \%)$ and ACPA $(75 \%)$ patients keep on reduced dose regimen.

Conclusions: We have deintensificated 62 out of 271 RA patients on BT (23\%). All patients were in clinical remission at the beginning of BT dose reduction for more than 6 months.

Most patients $(65 \%)$ analyzed remain long time with reduced BT in clinical remission.

We have not observed significant X-ray progression for reduced patients, even if they increase disease activity and need to back to the original BT dose.

The increase in disease activity was the main reason to interrupt the optimization regime.

Infliximab was the drug that remained more time optimized.

According to the results of our study, male patients negative for ACPA and FR remain longer with reduced doses

Disclosure of Interest: None declared

DOI: 10.1136/annrheumdis-2017-eular.5273

\section{AB0385 STRATEGIES FOR THE OPTIMIZATION OF BIOLOGICAL THERAPY IN PATIENTS WITH JOINT INFLAMMATORY DISEASE: ANALYSIS OF CLINICAL RESULTS AT 4 YEARS}

M.J. Rodriguez Valls, M.D. Toledo Coello, Y. Cabello Fernandez, M.V. Perez Romera, J.J. Perez Venegas. Reumatologia, Hospital de Jerez, Jerez de la Frontera, Spain

Background: Biological therapies optimization in joint inflammatory diseases is indicated in patients who have more than six months in clinical remission. The main objective is to limit the occurrence of adverse effects, which are dosagedependent. In addition, the cost savings suppose a better access of new patients to these treatments. Given the increasing importance of this topic in recent years, we present the optimization experience in our center at the last 4 years

Objectives: 1.To analyze the clinical evolution evaluated by DAS28 in patients with rheumatoid arthritis (RA) and polyarticular psoriatic arthritis (PPSA) in biological therapy (BT) followed in a university hospital in the south of Spain which are performed in optimization of BT. 2. To analyze the optimization strategies used with the different $\mathrm{BT}$

Methods: Observational, longitudinal, retrospective and descriptive study by the review of clinical records, between January 2013 and January 2017, of patients with RA and PPSA who underwent BT optimization for more than 6 months at follow-up (DAS28 <2.6) or minimal activity (DAS28 2.6-3.2). We analyze demographic data, time of evolution of illness before the use of BT, time of TB until optimization, clinical evaluation by DAS28 and therapeutic strategies. Statistical analysis was performed with the IBM SPSS Statistics program

Results: From 294 patients in BT (174 RA and 120 PPsA), 95 (32.3\%) were submitted to optimization treatment: 58 in RA group and 37 in PPSA group. 57 were women and 38 men, with $56 \pm 12$ years mean age. The mean treatment time at optimized doses was $32 \pm 17$ months. At the optimization time, 85 (89.5\%) patients presented DAS28 remission and $10(10.5 \%)$ had low activity; at the study cut time, $67(70.5 \%)$ of them continue at clinical remission, $14(14.7 \%)$ low activity, $11(11.6 \%)$ moderate and $3(3.2 \%)$ high activity according to DAS28. A $69.5 \%$ (66) of the patients continued with optimized doses at the end of the study. From the 58 patients included in the RA group, 14 ( 5 with adalimumab $40 \mathrm{mg} / 21$ days, 8 with etanercept $25 \mathrm{mg} / 7-10$ days or $50 \mathrm{mg} / 10$ days and 1 with certolizumab $200 \mathrm{mg} / \mathrm{month}$ ) need the standard dose of the drug for disease control; a patient receiving etanercept discontinued the treatment after the diagnosis of breast cancer, as well as three other patients treated with adalimumab who were diagnosed of pancreatic cancer, septic arthritis and cognitive impairment respectively. On the other hand, 9 patients from of the PPSA group needed to restart the treatment at standard doses, either for articular (4) or cutaneous (5) worsening, 3 of them being treated with adalimumab and 6 with etanercept. In addition, a patient had to be discontinued due to the diagnosis of decompensated liver cirrhosis; another patient suspended it voluntarily

Conclusions: After 4 years, we can conclude that in our cohort of patients with inflammatory joint disease in BT that have been submitted to dose optimization because they were in remission or low disease activity, a high percentage $(69.5 \%)$ remain in the same clinical situation for an average of 32 months. The response rate obtained for optimization in the RA group and in the PPSA group are comparable. The most frequent optimization strategies employed were adalimumab $40 \mathrm{mg} / 21$ days, etanercept $25 \mathrm{mg} / 7$ days and etanercept $50 \mathrm{mg} / 10$ days.

Disclosure of Interest: None declared DOI: 10.1136/annrheumdis-2017-eular.4326

\section{AB0386 A STUDY OF RETENTION AND SWITCHING RATES OF 1ST LINE BIOLOGICS FOR RHEUMATOID ARTHRITIS}

M. Sato, T. Tani, T. Ohashi. Rheumatology, Ohashi and Tani Orthopedics, Gifu, Japan

Background: Since the introduction of biologics for rheumatoid arthritis (RA) treatment, significant improvements in joint inflammation control, prevention of bone/joint destruction, activities of daily living ( $A D L)$, and quality of life (QOL) have been observed. Tumor necrosis factor (TNF) inhibitors, such as anti-TNF drugs and TNF receptor drugs, accounted for the majority of biologics. However, the use of non-TNF inhibitors, including interleukin (IL)-6 receptor antibodies and selective modulators of T-cell co-stimulation, has been approved, and their efficacy has been demonstrated.

Objectives: This study aimed to analyze the effective usage retention rate of TNF inhibitors and non-TNF inhibitors in RA patients.

Methods: Among 475 RA patients who were administered biologics up to the end of 2015, 329 patients who had been treated for more than 5 years (treatment initiated before the end of December 2010) since the first introduction of biologics (1st line) were included in this study. These patients were divided into TNF inhibitor (infliximab: IFX, etanercept: ETN, and adalimumab: ADA) and non-TNF inhibitor groups (tocilizumab: TCZ, and abatacept: ABT) to investigate the number of patients who progressed to 2 nd or 3 rd line therapy by 5 years after introduction, and which biologic was administered as the 2nd or 3rd line therapy in each group. Results: Of 329 patients, 67 were men and 262 were women. Patient age ranged between 22 and 83 years with a mean of $58.7 \pm 13.5$ years. RA disease duration ranged between 1 and 50 years with a mean of $9.6 \pm 8.1$ years. TNF inhibitors and non-TNF inhibitors were used as 1st line therapy in 278 and 51 patients respectively. In the TNF inhibitor group, IFX, ETN, and ADA were administered to 145,87 , and 46 patients respectively. In the non-TNF inhibitor group, TCZ and ABT were administered to 48 and 3 patients respectively. In the TNF inhibitor group, 94 of 278 patients (33.8\%) progressed to 2 nd line therapy owing to efficacy attenuation and adverse events. Thirty-four of these patients were switched to TNF inhibitors and 60 to non-TNF inhibitors. Conversely, 6 of 51 patients in the non-TNF inhibitor group (11.8\%) advanced to 2nd line therapy, with 2 switching to TNF inhibitors and 4 to non-TNF inhibitors. Additionally, 25 and 3 patients advanced to 3rd line therapy in the TNF inhibitor and non-TNF inhibitor groups respectively. Throughout the 5 years, 154 (55.4\%) patients in the TNF inhibitor group did not change their treatment agent while $69(24.8 \%), 24(8.6 \%)$, and $1(0.4 \%)$ switched once, twice, or 3 times or more, respectively. Thirty $(10.8 \%)$ patients discontinued biologic usage. In the non-TNF inhibitor group, 3 (5.9\%), $2(3.9 \%)$, and $1(2.0 \%)$ patients switched once, twice, or 3 times, respectively. Six $(11.8 \%)$ patients discontinued biologic usage, and no change in treatment occurred in $39(76.5 \%)$ patients. The mean number of biologic agent switches per patient in each group over 5 years was 0.43 times in the TNF inhibitor group and 0.20 times in the non-TNF inhibitor group, indicating that the number was significantly lower in the non-TNF inhibitor group $(p=0.0032)$.

Conclusions: While the sample size was small and patient characteristics varied, it appears that non-TNF inhibitors are not inferior to TNF inhibitors as 1st line therapy biologics in terms of retention rate and number of switches to TNF inhibitors. Disclosure of Interest: None declared

DOI: 10.1136/annrheumdis-2017-eular.3356

\section{AB0387 COMPARISON OF ETANERCEPT IN MONOTHERAPY AND COMBINATION WITH SYNTHETIC DMARDS: DATA FROM ATTRA REGISTRY}

P. Horák ${ }^{1}$, M. Skácelová ${ }^{1}$, L. Szczuková ${ }^{2}$, A. Smržová ${ }^{1}$, J. Vencovský $^{3}$ J. Závada ${ }^{3}$, K. Pavelka ${ }^{3}$ on behalf of ATTRA registry Czech Republic. ${ }^{1}$ III Department of internal Medicine, Faculty of Medicine and Dentistry, University Olomouc, Olomouc; ${ }^{2}$ Institute of Biostatistics And analysis, Masaryk University Brno, Brno; ${ }^{3}$ Institute of Rheumatolgy, Charles University of Prague, Prague, Czech Republic

Background: Etanercept (ETN) is an established bDMARDs for therapy of rheumatoid arthritis and some other inflammatory diseases. In rheumatoid arthritis patients ETN could be given in combination therapy with sDMARDs as well as in monotherapy. 